\title{
Quantitative x-ray phase nanotomography
}

\author{
Ana Diaz, ${ }^{1, *}$ Pavel Trtik, ${ }^{2}$ Manuel Guizar-Sicairos, ${ }^{1}$ Andreas Menzel, ${ }^{1}$ Pierre Thibault, ${ }^{3}$ and Oliver Bunk ${ }^{1}$ \\ ${ }^{1}$ Paul Scherrer Institut, CH-5232 Villigen PSI, Switzerland \\ ${ }^{2}$ Empa, Swiss Federal Laboratories for Materials Science and Technology, CH-8600 Dübendorf, Switzerland \\ ${ }^{3}$ Department of Physics, Technische Universität München, D-85748 Garching, Germany
}

(Received 2 November 2011; revised manuscript received 9 January 2012; published 31 January 2012)

\begin{abstract}
$\mathrm{X}$-ray ptychographic computed tomography has recently emerged as a nondestructive characterization tool for samples with representative sizes of several tens of micrometers, yet offering a resolution currently lying in but not limited to the 100-nm range. Here we evaluate the quantitativeness of this technique using a model sample with a known structure and density, and we discuss its sensitivity as a function of resolution. Additionally, we show an example application for the determination of the mass density of individual 2- $\mu \mathrm{m}$-sized $\mathrm{SiO}_{2}$ microspheres with a relative error of $2 \%$. The accuracy and sensitivity demonstrated in this paper will enable quantitative imaging, segmentation, and identification of different phases in complex materials at the nanoscale.
\end{abstract}

DOI: 10.1103/PhysRevB.85.020104

PACS number(s): 68.37.Yz, 81.70.Tx, 87.57.Q-, 42.30.Rx

Quantitative three-dimensional (3D) mass density mapping at the nanoscale is relevant in many fields of research such as colloidal science, medicine, and the development of advanced materials. However, not many methods can provide a measurement of the mass density of a specimen with submicrometer resolution. Absorption contrast full-field x-ray microscopy can achieve 50-nm resolution. ${ }^{1}$ Yet $\mathrm{x}$-ray absorption contrast does not provide quantitative measurements of the electron density unless measurements at two different wavelengths are performed, ${ }^{2}$ and other $\mathrm{x}$-ray phase contrast modalities such as Zernike microscopy are quantitative only for specimens with small phase contrast and negligible absorption. ${ }^{3}$

Similarly to holotomography, ${ }^{4} \mathrm{x}$-ray coherent diffractive imaging (CDI) provides quantitative high-resolution phase images of a specimen with both phase and absorption contrast. ${ }^{5,6}$ Using phase retrieval algorithms, ${ }^{7}$ CDI reconstructs the complex wave front past the specimen from coherent diffraction patterns measured in the far field. Unlike other microscopy techniques, CDI does not require a lens to produce an image, and its resolution is only limited by the angular spread of the scattered intensity by the specimen. $\mathrm{X}$-ray ptychography ${ }^{8,9}$ has been developed as a variant of CDI in which a pinhole or a focusing element is used to define a confined and coherent illumination onto the sample, and diffraction patterns are recorded for overlapping regions of the specimen. The redundancy in the data arising from the overlapping areas allows the procedure to reconstruct simultaneously the specimen and the illumination function, ${ }^{10}$ providing a robust reconstruction of the complex wave field past the specimen. The combination of many ptychographic reconstructions at different incidence angles of the x-ray beam can be used for the tomographic rendering of the 3D electron density distribution of the specimen on the nanoscale. ${ }^{11}$

Compared to other CDI techniques, ptychography has the advantage of imaging extended samples of arbitrary size. ${ }^{8,9}$ This opens the possibility of quantitatively imaging representative volumes of a specimen at the nanoscale, which can be of relevance, for example, for simulations of hydration processes of cement on the nanoscale, ${ }^{12}$ or for the study of bone mineralization density distributions. ${ }^{13}$ The demonstration of the quantitativeness of the technique is a necessary step toward the realization of these relevant studies. Here we validate the quantitativeness of ptychographic tomography and we analyze its sensitivity as a function of resolution using a test object with known density and structure. We also demonstrate the application of the technique for the determination of the density of individual $\mathrm{SiO}_{2}$ microspheres with $2-\mu$ m diameter.

By solving the phase problem, ptychography retrieves the complex transmission function of the object, providing both amplitude and phase contrast. The phase is particularly relevant for quantitative imaging. It is defined as the shift of the incident wave-field phase as it passes through the object and can be expressed as

$$
\phi(x, y)=-\frac{2 \pi}{\lambda} \int \delta(\mathbf{r}) d z,
$$

where $\delta(\mathbf{r})$ is the difference from unity of the real part of the refractive index distribution within the object, $\lambda$ is the wavelength of the radiation, and $z$ is the propagation direction. A tomographic reconstruction from many projections such as Eq. (1) taken at different rotation angles of the sample with respect to the incident beam leads to the $3 \mathrm{D}$ distribution of the refractive index $\delta(\mathbf{r})$. Away from the absorption edges, the 3D electron density distribution can be readily obtained by

$$
n_{\mathrm{e}}(\mathbf{r})=\frac{2 \pi \delta(\mathbf{r})}{\lambda^{2} r_{0}},
$$

where $r_{0}$ denotes the classical electron radius. Therefore, $\mathrm{x}$-ray phase tomography provides directly the 3D electron density distribution of the specimen. The relation between electron density and mass density $\rho$ depends on the atomic species

$$
\rho=\frac{n_{\mathrm{e}} A}{N_{\mathrm{A}} Z},
$$

where $A$ is the molar mass, $Z$ is the total number of electrons in a molecule and $N_{\mathrm{A}}$ is Avogadro's number. It follows that knowledge of the chemical composition of the specimen allows an accurate measurement of the mass density distribution. ${ }^{14}$

Our experiments were performed at the cSAXS beamline at the Swiss Light Source, Paul Scherrer Institut, Switzerland, with $6.2-\mathrm{keV}$ radiation. We used a $2.5-\mu \mathrm{m}$ pinhole to define a coherent illumination onto the sample, which was placed 


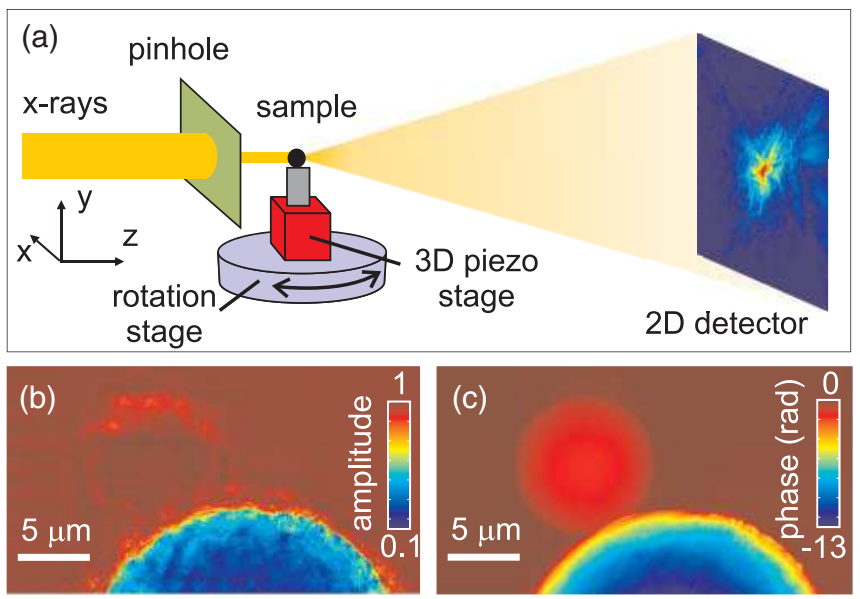

FIG. 1. (Color online) (a) Sketch of the experimental setup for ptychographic tomography. At each incidence angle coherent diffraction patterns are recorded and the projected complex transmission function can be reconstructed. Example of amplitude (b) and phase (c) of a single projection of a test sample consisting of two spheres.

$2.5 \mathrm{~mm}$ downstream of the specimen, as shown in Fig. 1(a). The sample was mounted on a 3D piezo stage in such a way that translations with nanometer resolution were possible in all directions. Below, a rotation stage was used to change the incident angle of the beam onto the sample over a range of $180^{\circ}$. Coherent diffraction patterns were acquired with a zero read-out noise and single-photon counting detector ${ }^{15}$ placed $7.2 \mathrm{~m}$ downstream. A He-filled flight tube in between reduced air scattering and absorption.

The first sample consisted of a 22.5- $\mu$ m-diam $\mathrm{BaTiO}_{3}$ sphere $^{16}$ and a $10-\mu$ m-diam polystyrene sphere ${ }^{17}$ attached on top. A layer of Pt of about $0.5-\mu \mathrm{m}$ thickness was deposited on a part of the $\mathrm{BaTiO}_{3}$ sphere surface, in which several letters were milled with a focused ion beam (FIB). The smallest features in the milled pattern were smaller than $200 \mathrm{~nm}$. The densities of the spheres were stated by the manufacturers to be $1.05 \mathrm{~g} / \mathrm{cm}^{3}$ for the polystyrene and $4.2 \mathrm{~g} / \mathrm{cm}^{3}$ for the $\mathrm{BaTiO}_{3}$. Ptychographic scans of the sample were performed in a circular grid $^{18}$ with a spacing of $1.5 \mu \mathrm{m}$ between the circular shells and a field of view of $30 \mu \mathrm{m} \times 20 \mu \mathrm{m}$ in the horizontal and vertical directions, respectively, resulting in a total of 213 points per scan. At each scanning point a coherent diffraction pattern was acquired with 0.4-s exposure time, spanning an angular range of $0.26^{\circ}$ on the detector. The total acquisition time required for a single projection was about $1.5 \mathrm{~min}$, which corresponded to a total of $6 \times 10^{8}$ photons incident on the sample, and estimated absorbed doses of 4 and $47 \mathrm{kGy}$ for the polystyrene and $\mathrm{BaTiO}_{3}$ spheres, respectively.

Ptychographic reconstructions with phase retrieval were performed using a difference map algorithm as described elsewhere. ${ }^{10}$ We show in Figs. 1(b) and 1(c) the reconstructed amplitude and phase, respectively, of one of the projections. The reconstruction pixel size was $43.6 \mathrm{~nm}$. The amplitude shows the upper part of the $\mathrm{BaTiO}_{3}$ sphere with a high contrast ranging from 1 in air to 0.1 in the thicker part of the sphere. The phase shows the same sphere with less noise and additionally reveals the polystyrene sphere, which is practically invisible
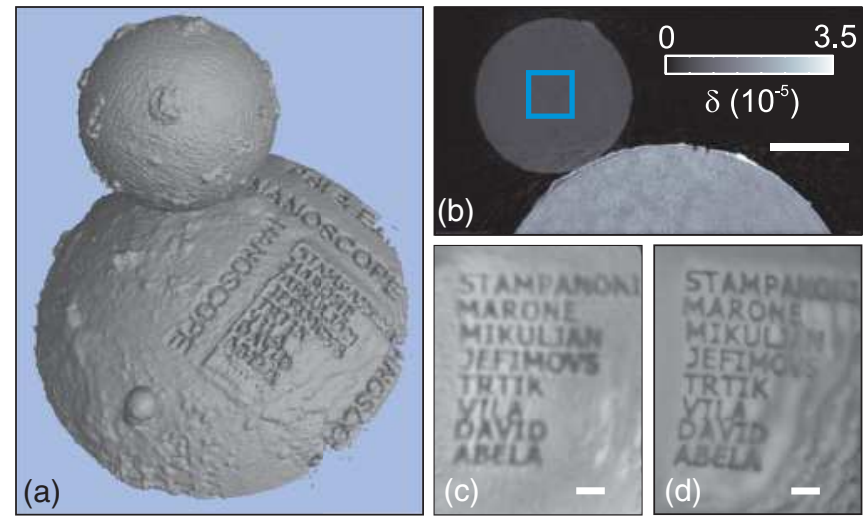

FIG. 2. (Color online) (a) 3D isosurface representation of the tomographic reconstruction of a test sample. (b) 2D slice of the tomogram in (a), scale bar $5 \mu \mathrm{m}$. (c) Spherical slice of the tomogram in (a) showing a region with milled letters on the surface of the sample. (d) SEM image of the same region shown in (c). Scale bars in (c) and (d) are $1 \mu \mathrm{m}$.

in absorption contrast. A phase range larger than $4 \pi$ and a zero value of the phase in air were obtained after phase unwrapping ${ }^{19}$ and removal of an offset. ${ }^{11,20}$ In the amplitude image we observe a significant amount of artifacts, as it has been reported in previous studies. ${ }^{11}$

Identical scans were recorded at 360 different incidence angles from $-90^{\circ}$ to $89.5^{\circ}$ in steps of $0.5^{\circ}$. After postprocessing and alignment ${ }^{20}$ the phase projections from all incidence angles were used in a filtered back projection tomographic reconstruction to obtain the 3D refractive index distribution $\delta(\mathbf{r})$ of the test sample. Figure 2(a) shows an isosurface representation of the measured $\delta(\mathbf{r})$ at a value close to the refractive index of polystyrene. On the surface of the large sphere we clearly observe the letters milled with the FIB. Figure 2(b) shows a two-dimensional (2D) slice through the reconstructed volume in a plane that passes close to the centers of the two spheres, where the gray scale indicates the refractive index. In this slice it is possible to resolve a high-density layer of a few hundred nanometer thickness on top of the $\mathrm{BaTiO}_{3}$ sphere arising from the $\mathrm{Pt}$ deposition. The resolution of the tomogram is about $150 \mathrm{~nm}$, estimated from a cut perpendicular to a sharp edge in the sample. This can be confirmed with a spherical slice of the $\mathrm{BaTiO}_{3}$ sphere at a region with milled structures, as shown in Fig. 2(c), where the milled letters are clearly resolved. In Fig. 2(d), a scanning electron microscopy (SEM) image shows the same region of the specimen at its surface, revealing a remarkable agreement with the tomographic data shown in Fig. 2(c).

To estimate the precision in the reconstructed density, we selected a volume of $64 \times 64 \times 64$ voxels in the polystyrene sphere, indicated by a square in Fig. 2(b), corresponding to a volume of approximately $(2.8 \mu \mathrm{m})^{3}$. The histogram of the reconstructed voxel values, shown in Fig. 3(a), fitted very well a Gaussian distribution, indicated by a line. The center of the distribution, $\delta_{0}=6.09 \times 10^{-6}$, and the standard deviation, $\sigma=0.29 \times 10^{-6}$, were used to determine the average electron density within the selected volume and its error by means of Eq. (2). We note that the standard deviation was very similar to the one obtained from a volume of identical size 

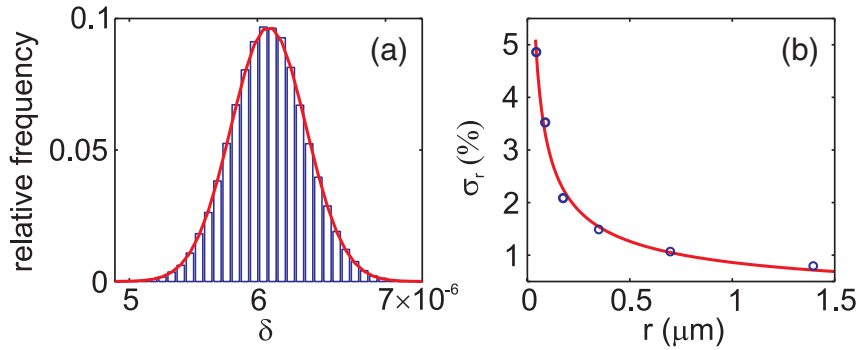

FIG. 3. (Color online) (a) Histogram of a region in the sample of approximately $(2.8 \mu \mathrm{m})^{3}$ size marked with a square in Fig. 2(b). (b) The circles show the relative error of the histogram $\sigma_{r}$ as a function of the voxel side length $r$, obtained by binning the voxels within this region. The line shows a fit to the data (see text for details).

within a region in air $\sigma^{\text {air }}=0.36 \times 10^{-6}$, confirming that the chosen area on the sphere is homogeneous. The knowledge of the chemical composition of this material revealed a mass density of $(1.05 \pm 0.05) \mathrm{g} / \mathrm{cm}^{3}$, which agreed very well with the expected mass density given by the manufacturer. The error of this measurement corresponds to a measurement on a single voxel, but binning the density in the selected volume reduces the spread of the distribution $\sigma$. This effect is shown in Fig. 3(b), where the relative error $\sigma_{r}=\sigma / \delta_{0}$ is plotted as a function of the voxel size. We observe that the error decreases as $r^{-0.55}$, as indicated by the fitted line. Assuming uncorrelated noise in the data, one would expect a faster decay of $\sigma$ as $r^{-1.5}$. The slower decay of the relative error in our measurement arises from correlated noise introduced by the filtered back projection reconstruction ${ }^{21}$ and from the ptychographic reconstructions. By choosing a binning of $32^{3}$ voxels, corresponding to a voxel size of approximately $(1.4 \mu \mathrm{m})^{3}$, the measured density on that region is $1.048 \pm 0.008 \mathrm{~g} / \mathrm{cm}^{3}$. This value actually provides a very close match with the value of the density of single polystyrene particles reported by Grover et al. ${ }^{22}$

An analysis on a volume of the same size within the $\mathrm{BaTiO}_{3}$ sphere revealed a histogram with a standard deviation $\sigma=$ $1.4 \times 10^{-6}$, significantly larger than the one found in air or in the polystyrene sphere. We attribute this to a lower degree of homogeneity within this sphere. Nevertheless, the average density within this volume was $4.14 \pm 0.10 \mathrm{~g} / \mathrm{cm}^{3}$ using a binning of $32^{3}$ voxels, in agreement with the value given by the manufacturer.

Ptychographic tomography has further been applied to measure the mass density of individual microspheres. In order to illustrate this application, we show in Fig. 4(a) a $3 \mathrm{D}$ tomogram of a sample of $\mathrm{SiO}_{2}$ microspheres of $2-\mu \mathrm{m}$ diameter ${ }^{23}$ confined in a $\mathrm{SiO}_{2}$ microcapillary. As expected, the tomogram revealed a homogeneous density within the particles, especially visible in a line across one of the microspheres, shown in Fig. 4(d). A quantitative analysis over a $1-\mu \mathrm{m}^{3}$ volume within this particle revealed a mass density of $(1.91 \pm 0.04) \mathrm{g} / \mathrm{cm}^{3}$, and similar values have been obtained for the other particles in the sample. The measured values were significantly smaller than expected for bulk $\mathrm{SiO}_{2}$. Silica microspheres can have significantly different densities than their corresponding bulk materials, and it is usually not straightforward to determine the exact mass density when

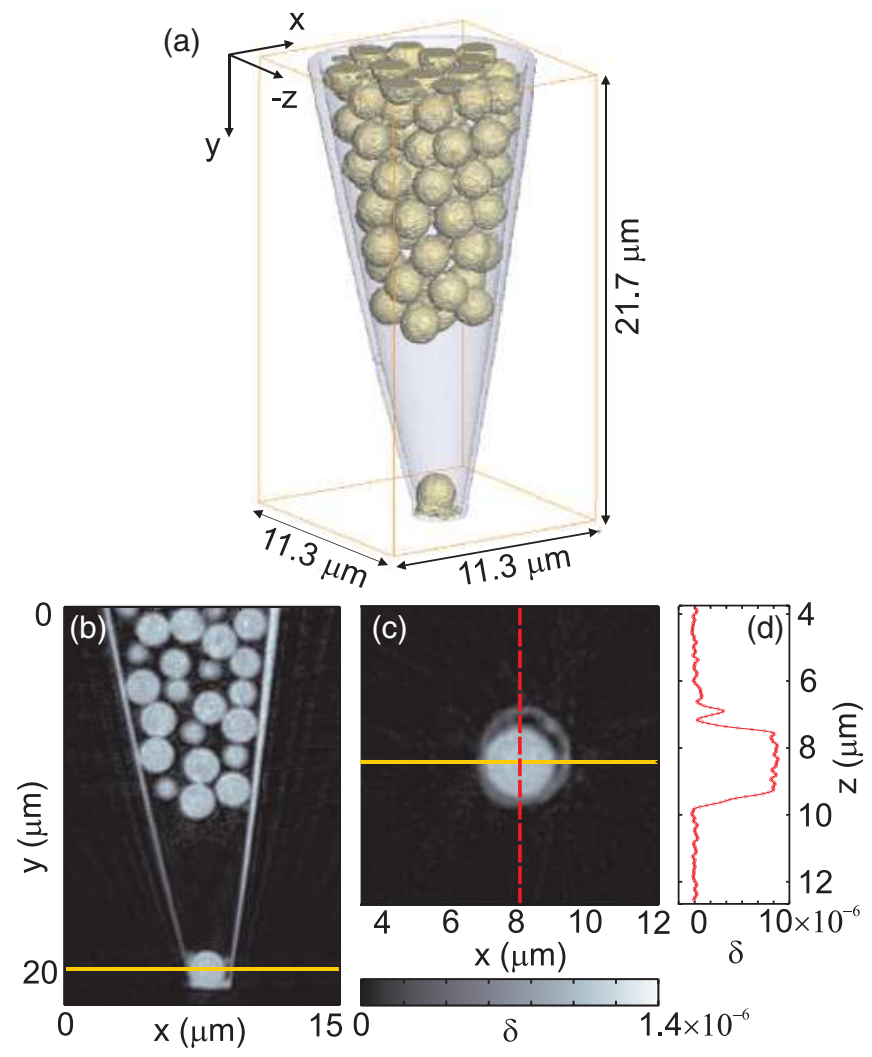

FIG. 4. (Color online) (a) 3D rendering of the ptychographic tomogram of a sample consisting of 2- $\mu$ m-diam $\mathrm{SiO}_{2}$ microspheres in a glass capillary. We show in yellow (dark gray) an isosurface representation of the microspheres, while the capillary is shown in a semitransparent light gray color. (b) 2D section through the tomogram shown in (a). (c) 2D section of the same tomogram perpendicular to the section shown in (b) at the position of the line. (d) Line profile indicated by a dashed line in (c) reveals the refractive index distribution within a particle and the capillary wall.

limited amounts of sample are available. ${ }^{24}$ Even though the recently developed techniques based on the nanomechanical resonators ${ }^{25}$ used for the assessment of the densities of single cells and single particles ${ }^{22}$ currently exhibit density resolution superior to ptychographic tomography, they are intrinsically limited to the provision of densities of the entire entities. Therefore, these techniques, unlike ptychographic tomography, cannot provide the internal density maps, which will prove very useful for the assessment of the phases of multicomponent particles, such as core-shell colloidal spheres.

In conclusion, we have shown that ptychographic tomography yields accurate quantitative mass density values whenever the specimen's chemical composition is known. The sensitivity of the density measurements has also been studied as a function of the binning of the 3D data, which can decrease statistical errors down to $1 \%$ in homogeneous samples. We have further demonstrated the technique as a method to measure the mass density of $\mathrm{SiO}_{2}$ microspheres with a relative error of $2 \%$. In this study the resolution is limited to 150 $\mathrm{nm}$ by mechanical stability and thermal drifts. However, the resolution is fundamentally limited only by the wavelength and the detector angular extent. In practice, other limiting factors are an effective depth of focus related to the specimen's 
thickness,,${ }^{9,26}$ and multiple scattering effects, also related to the specimen's thickness. ${ }^{27}$ With the coherent flux produced at third-generation synchrotron sources, and a dedicated nanotomography sample stage with improved mechanical stability, we believe that $(20 \mathrm{~nm})^{3}$ resolution for specimens up to $100-\mu \mathrm{m}$ size will be possible in the near future. The method of quantitative tomography described here requires that the field of view at each projection is larger than the lateral extension of the specimen and that the full angular range from $0^{\circ}$ to $180^{\circ}$ is accessible. For cases where these conditions are not fulfilled, other methods for quantitative tomographic reconstructions should be investigated and validated.

Our results demonstrate the feasibility and reliability of high-resolution measurements of density distributions with a resolution below $200 \mathrm{~nm}$, even in cases where representative sample volumes exceed many thousands of cubic micrometers. Such measurements already provide unique insight into advanced materials such as carbon fibers, where it can be crucial to determine graphite distribution within the bulk carbon matrix, both differing in mass density, but not in chemical composition. The method's accuracy, as demonstrated in this paper, facilitate highly specific segmentations in granular media even if different constituents of phase display a large degree of similarity. We expect this method of quantitative measurement of density distributions to find many applications in life and materials science.

We would like to thank X. Donath and J. Krbanjevic for technical support and M. Dierolf, M. Stampanoni, and F. Marone for fruitful discussions.

*ana.diaz@psi.ch

${ }^{1}$ M. A. Le-Gross, G. McDermott, and C. A. Larabell, Curr. Opin. Struct. Biol. 15, 593 (2005).

${ }^{2}$ M. Torikoshi, T. Tsunoo, M. Sasaki, M. Endo, Y. Noda, Y. Ohno, T. Kohno, K. Hyodo, K. Uesugi, and N. Yagi, Phys. Med. Biol. 48, 673 (2003).

${ }^{3}$ U. Neuhäusler, G. Schneider, W. Ludwig, M. A. Meyer, E. Zschech, and D. Hambach, J. Phys. D 36, A79 (2003).

${ }^{4}$ G. Requena, P. Cloetens, W. Altendorfer, C. Poletti, D. Tolnai, F. Warchomicka, and H. P. Degischer, Scr. Mater. 61, 760 (2009).

${ }^{5}$ J. Miao, P. Charalambous, J. Kirz, and D. Sayre, Nature (London) 400, 342 (1999).

${ }^{6}$ H. N. Chapman and K. A. Nugent, Nat. Photonics 4, 833 (2010).

${ }^{7}$ J. R. Fienup, Appl. Opt. 21, 2758 (1982).

${ }^{8}$ J. M. Rodenburg, A. C. Hurst, A. G. Cullis, B. R. Dobson, F. Pfeiffer, O. Bunk, C. David, K. Jefimovs, and I. Johnson, Phys. Rev. Lett. 98, 034801 (2007).

${ }^{9}$ P. Thibault, M. Dierolf, A. Menzel, O. Bunk, C. David, and F. Pfeiffer, Science 321, 379 (2008).

${ }^{10}$ P. Thibault, M. Dierolf, O. Bunk, A. Menzel, and F. Pfeiffer, Ultramicroscopy 109, 338 (2009).

${ }^{11}$ M. Dierolf, A. Menzel, P. Thibault, P. Schneider, C. M. Kewish, R. Wepf, O. Bunk, and F. Pfeiffer, Nature (London) 467, 436 (2010)

${ }^{12}$ D. P. Bentz, Mater. Struct. 40, 397 (2007).

${ }^{13}$ P. Roschger, E. P. Paschalis, P. Fratzl, and K. Klaushofer, Bone 42, 456 (2008).

${ }^{14}$ We note that $\rho$ is mostly dependent on $n_{\mathrm{e}}$, since the ratio $A / Z \approx$ $2 \mathrm{~g}$ for most atomic species except for H. However, knowledge of the chemical composition of the specimen is often needed for an accurate determination of $\rho$.

${ }^{15}$ P. Kraft, A. Bergamaschi, C. Brönnimann, R. Dinapoli, E. F. Eikenberry, H. Graafsma, B. Henrich, I. Johnson, M. Kobas, A. Mozzanica, C. M. Schlepütz, and B. Schmitt, IEEE Trans. Nucl. Sci. 56, 758 (2009).

${ }^{16}$ Microspheres-Nanospheres, Corpuscular Inc., custom-made sample.

${ }^{17}$ Interfacial Dynamics Corporation, product number 1-10000.

${ }^{18}$ M. Dierolf, P. Thibault, A. Menzel, C. M. Kewish, K. Jefimovs, I. Schlichting, K. von König, O. Bunk, and F. Pfeiffer, New J. Phys. 12, 035017 (2010)

${ }^{19}$ R. M. Goldstein, H. A. Zebker, and C. L. Werner, Radio Sci. 23, 713 (1988).

${ }^{20}$ M. Guizar-Sicairos, A. Diaz, M. Holler, M. S. Lucas, A. Menzel, R. A. Wepf, and O. Bunk, Opt. Express 19, 21345 (2011).

${ }^{21}$ S. J. Riederer, N. J. Pelc, and D. A. Chesler, Phys. Med. Biol. 23, 446 (1978).

${ }^{22}$ W. H. Grover, A. K. Bryan, M. Diez-Silva, S. Suresh, J. M. Higgins, and S. R. Manalis, Proc. Natl. Acad. Sci. U.S.A. 108, 10992 (2011).

${ }^{23}$ Microspheres-Nanospheres, Corpuscular Inc., product number 147114-10.

${ }^{24} \mathrm{~J}$. Lyklema, Fundamentals of Interface and Colloid Science ( Elsevier, Amsterdam, 2005), Vol. IV.

${ }^{25}$ T. P. Burg, M. Godin, S. M. Knudsen, W. Shen, G. Carlson, J. S. Foster, K. Babcock, and S. R. Manalis, Nature (London) 446, 1066 (2007).

${ }^{26}$ J. M. Rodenburg and R. H. T. Bates, Philos. Trans. R. Soc. London A 339, 521 (1992)

${ }^{27}$ C. Liu, T. Walther, and J. M. Rodenburg, Ultramicroscopy 109, 1263 (2009). 